\title{
Review Article \\ The Role of Galectins in Cervical Cancer Biology and Progression
}

\author{
Lufang Wang, Yanyan Zhao, Yanshi Wang, and Xin Wu \\ Department of Gynecology, The First Affiliated Hospital of China Medical University, Shenyang, Liaoning, China \\ Correspondence should be addressed to Xin Wu; xinwu.1964@aliyun.com
}

Received 9 December 2017; Revised 18 March 2018; Accepted 27 March 2018; Published 8 May 2018

Academic Editor: Steven De Vleeschouwer

Copyright (C) 2018 Lufang Wang et al. This is an open access article distributed under the Creative Commons Attribution License, which permits unrestricted use, distribution, and reproduction in any medium, provided the original work is properly cited.

\begin{abstract}
Cervical cancer is one of the malignant tumors with high incidence and high mortality among women in developing countries. The main factors affecting the prognosis of cervical cancer are the late recurrence and metastasis and the effective adjuvant treatment, which is radiation and chemotherapy or combination therapy. Galectins, a family containing many carbohydrate binding proteins, are closely involved in the occurrence and development of tumor. They are involved in tumor cells transformation, angiogenesis, metastasis, immune escape, and sensitivity against radiation and chemotherapy. Therefore, galectins are deemed as the targets of multifunctional cancer treatment. In this review, we mainly focus on the role of galectins, especially galectin-1, galectin-3, galectin-7, and galectin-9 in cervical cancer, and provide theoretical basis for potential targeted treatment of cervical cancer.
\end{abstract}

\section{Introduction}

As the second most common female malignant tumor around the world, cervical cancer occupies the second place of fatality in gynecological oncology in developing countries [1]. The present studies showed that cervical cancer is closely associated with the infection of high risk human papillomavirus (HPV), but there are still a large number of patients with cervical cancer who are not infected with HPV, suggesting that other factors such as cell genetic changes can also lead to disease progression [2]. The main treatments for cervical cancer are surgery (including pelvic lymphadenectomy and radical hysterectomy), radiotherapy, and chemotherapy. Both radical hysterectomy and radiotherapy are considered curative for localized disease, while for advanced cancer, concurrent radiochemotherapy remains a cornerstone intervention [3]. Targeted therapy is becoming a hot spot of research in recent years and the current clinical targeted therapies primarily for the treatment of cervical carcinoma are EGFR $[4,5]$ and COX-2 $[6,7]$. However, the survival rate of cervical cancer did not increase significantly but increased with adverse events. Therefore, further prognosis biomarkers and therapeutic targets research need to be carried out.
Galectins, a family of $\beta$-galactoside binding proteins, widely exist not only in animals but also in bacteria and fungi at different levels. They are a highly conserved core sequence containing 130 amino acids and a carbohydrate recognition domain (CRD). There are two typical characteristics of galectins protein family: (1) sharing significant similarities in a conserved amino acid sequence; (2) a high affinity for beta galactoside sugars [8]. It has been discovered and named that galectins (Gals) in mammals have 15 subtypes. According to their molecular structure, they are divided into three types: (1) prototype galectins: prototype galectins are proteins containing a single carbohydrate recognition domain (CRD), which often forms homodimers and includes galectin1, galectin-2, galectin-5, galectin-7, galectin-10, galectin-11, galectin-13, galectin-14, galectin -15; (2) chimeric galectins: chimeric galectins contain two domains, one C-terminal CRD and one noncarbohydrate-binding $\mathrm{N}$-terminal domains self-associating into oligomers, and only include galectin3; (3) tandem-repeat galectins: tandem-repeat galectins are dimers consisting of two CRDs connected by a linker peptide and include galectin-4, galectin-6, galectin-8, galectin-9, galectin-12 [9] (Figure 1). Galectins are reported to have multiple roles in different parts of the cells, such as cell 


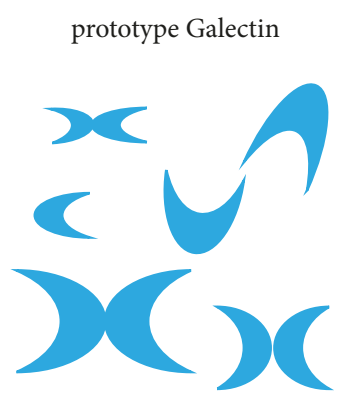

Galectin-1, -2, -5, -7, -10,-11, $-13,-14,-15$.

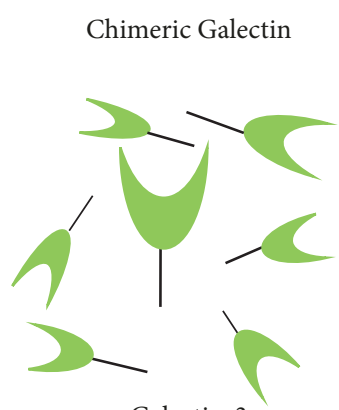

Galectin-3

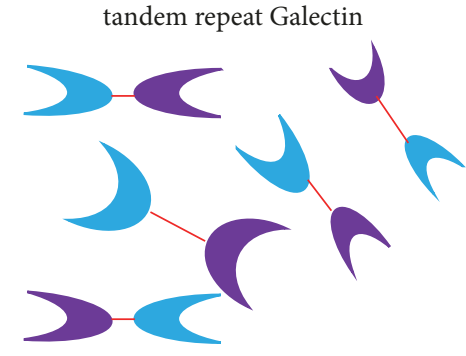

Galectin- $4,-6,-8,-9,-12$

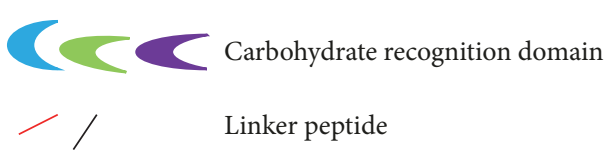

FIGURE 1: Galectins are divided into three types according to their molecular structure: (1) prototype galectin (galectin-1, galectin-2, galectin-5, galectin-7, galectin-10, galectin-11, galectin-13, galectin-14, and galectin-15); (2) chimeric galectin (galectin-3); and (3) tandem repeat galectin (galectin-4, galectin-6, galectin-8, galectin-9, and galectin-12).

membrane, cytoplasm, extracellular matrix, and the intracellular receptors which are numberous, such as cytokeratins, cyclins, transcription factors, Bcl-2, and h-rask-ras [10, 11]. Galectins can be secreted into extracellular matrix [12]. Since galectins lack signal peptide, they can only be synthesized in the cytoplasm and then secreted to the outside of the cell by the vesicles directly, the endoplasmic reticulum and Golgi apparatus [13]. The special way of secretion can prevent galectins from adhering to new generation of glycoprotein oligosaccharide prematurely [11]. Some factors affect the secretion of galectins, such as components of the extracellular matrix and inflammatory factors [14]. Galectins on the cell surface, the secretion of extracellular matrix, and cellular and extracellular are closely related to cell adhesion and signal transduction [15]. Galectins outside the cells combine to diverse cell surface receptors forming as carbohydrates $[16,17]$. Therefore, not only in the aspects of tumorigenesis and development, but also in terms of organogenesis and connective tissue diseases, galectins all play an important role [18].

Many previous experimental researches showed that galectins play an essential role in the origin and development of cancer, such as angiogenesis [19], cell adhesion, invasion, and migration [20]. However, the role and mechanism of the same galectin is different in different tumors. For example, upregulated Gal-3 contributed to increased cancer cell migration and motility through downregulating K-Ras-RafErk1/2 pathway in colon cancer $[21,22]$. In gastric cancer, Gal-3 may promote metastasis by enhancing the expression of MMP-1 and protease-activated receptor-1 (PAR-1) [23]. In vitro as well as in vivo studies had indicated tumor suppressive effect of galectin-7 on colon cancer [24]. Even in the same kind of tumor, the role of different galectins is not also the same; for example, galectin-1 is overexpressed and can increase metastasis of colorectal cancer [25] while Gal-4 is downregulated in colorectal cancer [26]. Gordower et al. found that the level of Gal-3 expression significantly decreases in astrocytic tumors from low grade to high grade while some highly malignant tumor cells increase expressed higher than normal tissue [27]. Studies showed that the expression of galectins changes a lot in cervical cancer [28, 29]. But there is no review on the relationship between cervical cancer and galectins. In the review, we focus on the biological role of galectins in the development of cervical cancer and their potential role in targeted therapy of cervical cancer.

\section{Galectins in Cancer}

The activation of protooncogenes to the occurrence and development of cancer entails a lot of complex processes that involves multiple factors. These factors are dependent on genetic changes, external cellular pressures, function and regulation of the body's immune system, and the microenvironment of the tissue [30]. Studies showed that most galectins are involved in the development of various cancers [31, 32] and the processes they involved in are mainly tumor cell transformation through interacting with oncogenes such as HRAS and KRAS $[33,34]$. It has been found that galectins not only participate in cell cycle and cell apoptosis [35] but also are involved in the development of tumor through tumor immune escape, tumor metastasis, and tumor angiogenesis [36].

2.1. The Role of Galectins in Cancer Cell Proliferation. The proliferation and metastasis of malignant tumor cells are the important factors that influence the treatment of tumor. Therefore, understanding the factors and mechanism that influence and regulate malignant tumor cells proliferation and metastasis is of great significance for early diagnosis and clinical treatment of tumors. 
TABLE 1: Effect of galectins on tumor biology and treatment.

\begin{tabular}{lcccc}
\hline Process & Galectin-1 & Galectin-3 & Galectin-7 & Galectin-9 \\
\hline Proliferation & $\uparrow$ & $\uparrow$ & $\downarrow$ & $\uparrow$ \\
Apoptosis & $\uparrow$ & $\downarrow$ & $\uparrow$ & $\uparrow$ \\
Metastasis: invasion and adhesion & $\uparrow$ & $\uparrow$ & $\downarrow$ & $\downarrow$ \\
Tumor angiogenesis & $\uparrow$ & $\uparrow$ & $\downarrow$ & $\downarrow$ \\
Immune responses & $\downarrow$ & $\downarrow$ & $\downarrow$ & UNK \\
Radiotherapy and/or chemotherapy resistance & $\uparrow$ & $\uparrow$ & $\downarrow$
\end{tabular}

UNK, unknown; $\uparrow:$ increase; $\downarrow$ : decrease.

In tumor cell experiments, overexpression of Gal-1 will stimulate cell proliferation in human glioma cells [37] and thyroid cancer [38]. Mercier et al. [39] showed that Gal1 mainly regulates cell cycle through Ras/Raf/ERK2 signaling pathway. Gal-3 also plays a tumor promotion role in the proliferation of cancers cells. Gal-3 can increase cell growth of hepatocellular carcinoma [40], glioma cells [41], pancreatic cancer proliferation, The effects of Gal-3 on the cell proliferation and cycle came about as a result of the translocation ability of Gal-3 into the nucleus by binding with Impotin, Sufu, and Nup98, wherein it controls the cell cycle through the interaction with cyclin A, cyclin D, cyclin E, p21 (WAF1), and p27 (KIP1), accelerating cancer cell proliferation [42]. Studies performed on diverse cell types, mostly cancerous cell lines, have demonstrated that galectin7 has a suppressive effect on cell proliferation. Indeed, ectopic expression or addition of exogenous galectin-7 in the DLD1 human colon carcinoma cell line [24] and the neuroblastoma cells SK-N-MC, respectively [43], drastically reduced tumor cell proliferation. Studies performed on diverse cell types, mostly cancerous cell lines, have demonstrated that galectin-7 has a suppressive effect on cell proliferation. The molecular mechanism by which galectin-7 participates in cell proliferation remains to be clarified. However, galectin7 could be an effector of the tumor suppressor gene p53. Strikingly, galectin-7 expression is strongly induced by $\mathrm{p} 53$ [44] and lack of wild type p53 in human keratinocytes cell lines prevents galectin-7 expression induction in response to UVB irradiation [45]. Gal-9 can induce cell proliferation in human osteoblasts through sc-Src-ERK and NFkB signals pathway [46] (Table 1).

2.2. The Role of Galectins in Cancer Cell Apoptosis. Cancer is a disease with abnormal cell proliferation and cell death. Solid tumors cells mainly have two death forms, necrosis or apoptosis. Cell apoptosis not only plays a significant role in tumorigenesis and development but comes to play when chemotherapy, radiotherapy, and biological therapy are used to treat cancers [47]. One way to treat cancer is to control or possibly stop the uncontrolled growth of cancer cells. One way to treat cancer is to control or possibly stop the uncontrolled growth of cancer cells. Since apoptosis avoidance is a characteristic of cancer, targeted apoptosis may become the most successful nonsurgical treatment. Nowadays, many antitumor drugs are aimed at different endogenous and exogenous pathways [48-50]. Two commonly used therapeutic targeting strategies are stimulating apoptotic molecules and inhibiting antiapoptotic molecules [51]. Some of the targets that have been researched include ligands for deathreceptors, XIAP inhibition [52], inhibitors for BCL-2 [53], and alkylphospholipid analogs (APL) which act as apoptotic signals [49]. Studies showed that Gal-1 binding induces apoptosis through triggering apoptotic pathways (induction of the activation protein-1 (AP-1) transcription factor and then the activation of apoptotic pathway promoters caspase- 8 and caspase- 3 and subsequently downregulation of mitochondrial $\mathrm{Bcl}-2$ ) [54] and promoting TCR chain phosphorylation [55]. On the contrary, Gal-3 can suppress cells apoptosis and the antiapoptotic activity is given by a functional antideath (NWGR) motif, an conserved amino acid sequence located in the $\mathrm{BH} 1$ domain of the $\mathrm{Bcl} 2$ gene family [56]. Gal-3 combines with CD95/Fas and subsequently suppresses the activation of CD95/Fas-mediated caspase8 [57]. The inhibitory effect of Gal-3 on cell apoptosis is regulated by the phosphorylation/dephosphorylation of ser- 6 residues, which acts as a "switch" in binding Gal-3 to ligands [58]. Gal-7 and Gal-9 tend to display proapoptotic effects. Moisan et al. [59] have shown that Gal-7 plays a promoter role in tumor cells apoptosis through activating mitochondrial cytochrome c release and JNK activity. Gal-9 also induces apoptosis through activating JNK and p38-MAPK pathway and subsequently activating caspase-3, caspase-8, and caspase-9 in multiple myeloma cells [60]. (Table 1).

2.3. Metastasis: Invasion and Adhesion. Tumor invasion and metastasis are the unique characteristics of malignant tumor compared with benign tumor and they are also the most important factors that affect treatment and prognosis. There are many essential steps that carry out cancer cells metastasis. Firstly, the connection between the cells became loose; cancer cells invade to extracellular matrix through interactions among cells and adhesion to vascular endothelium. Then the invaded cancer cells moved to distant sites through hemokinesis and proliferate by forming new blood vessels and then transfer to other place [61]. The cytokines involve tumor endothelial cell interactions that occur between various surface adhesion molecules including integrins, ICAM-1, VCAM-1, or selectins [62]. Studies showed that Gal-1 binding with CD44 and CD326 promotes tumor cells metastasis to cell matrix and adhesion to vascular endothelial cells 
and knockdown of Gal-1 significantly reduced their lung metastatic potential in colon and breast cancer $[63,64]$. Merseburger et al. found that Gal-3 was highly expressed in clear cell renal carcinoma (CC-RCC) especially in those patients with distant metastasis [65]. O'Driscoll et al. confirmed that overexpression of Gal-3 can increase the adhesion between lung cancer cells to extracellular matrix, leading to cell motility and invasiveness, and inhibiting Gal-3 by lactose will significantly weaken this effect [66]. Matarrese et al. found that Gal-3 promotes invasiveness of human breast carcinoma cells through interaction with the $\alpha 6 \beta 1$ integrin [67]. Iurisci et al. [68] also showed that higher Gal-3 in blood of cancer patients could induce the vascular endothelial cells to secrete more cytokines like colony-stimulating factor (G-CSF) and interleukin-6 (IL-6), leading to interactions among the cytokines, vascular endothelial and cancer cells, and ultimately increasing endothelial cells migration and tubule formation. Demers et al. [69] showed that through regulating metastatic genes like metalloproteinase 9 (MMP9) Gal-7 suppresses the invasion of lymphoma cells. Gal-9 can inhibit invasion and metastasis of tumor cells and is a protective factor affecting prognosis. Transfected with Gal9 in colon cancer and melanoma models can significantly decrease the risk of metastasis [70]. Kageshita et al. [71] also confirmed that downregulation of gal-9 in hepatocellular carcinoma cells (HCC) could significantly suppress the risk of lymph node metastasis, vascular invasion, and intrahepatic metastasis, improving survival rate of patients. They also analyzed how Gal-9 inhibited invasion and metastasis of HCC and showed that Gal-9 blocked cancer cells adhesion to extracellular matrix (ECM) through downregulation of ECM components such as collagen, laminin, and fibronectin (Table 1).

2.4. The Role of Galectins in Tumor Angiogenesis. Angiogenesis plays an important role for the development and evolution of tumor, since the rapid expansion of tumor requires continuous new blood vessels, which is very important for the growth and metastasis of tumor cells. Ito et al. [72] reported that adding exogenous Gal-1 increases the capillary-like tube forming capacity of endothelial cells within basal membrane Matrigel cultures. The extracellular Gal-1 structurally promotes tumor angiogenesis by reinforcing and stabilizing connections of vascular endothelial cells and extracellular matrix interactions within the tumor microenvironments. Studies showed that Gal-1 can induce angiogenesis and thiodigalactoside can block effects of Gal-1 through suppressing angiogenesis and protection against oxidative stress. As a chemoattractant inducting epithelial cells transfer to vascular endothelial cells in vitro and in vivo, Gal-3 is critical for tumor angiogenesis [73]. Markowska et al. [74] found that Gal-3 is involved in VEGF- and bFGF-mediated angiogenesis and through binding with GnTV-modified N-glycans on $\alpha \mathrm{v} \beta 3$ integrin, they activate FAK-mediated signaling pathways which modulate endothelial cell migration in the angiogenic cascade. Their further study [75] showed that Gal-3 also binds to VEGFR2 to increase angiogenic response to VEGFA. Another study showed that the combined action of Gal1 and Gal-3 can increase effect on angiogenesis via the activation of VEGFR1 and the decrease receptor endocytosis [76]. Gal-9 appears to be the only galectin with an inhibitory effect on angiogenesis. It remains to be established which mechanisms underlie this inhibitory effect [77]. Thus far, the angiostimulatory activity of galectins has been linked to several signaling pathways, including the VEGF/VEGFR2 signaling axis, integrin signaling, and Ras signaling $[78,79]$ The ability of galectin-1, galectin-3, and galectin- 8 to trigger these signaling pathways has been linked to cross-linking of different receptors like VEGFR2, neuropilin-1, beta-integrins, and CD166 [80]. Whether galectin-9 also cross-links these receptors on endothelial cells remains to be established. In fact, most of the receptors found to interact with galectin- 9 appear to be predominantly involved in immune cell activity and function, for example, T-cell immunoglobulin mucin 3 (TIM-3), cell surface bound protein disulfide isomerases (PDI), CD40, and CD44 [81]. Interestingly, since several of these receptors are also expressed by endothelial cells, it could be hypothesized that some are involved in regulating the effects of galectin-9 in angiogenesis (Table 1).

2.5. The Role of Galectins in Immune Responses. In the traditional sense, the immune cells play an important role of monitoring the occurrence of tumor through a series of mechanisms from the host immune attack. T lymphocytes, natural killer (NK) cells and cytokines induced Killer cells (CIK), dendritic cells (DC) and DC-CIK cells, and so on all have certain antitumor activity but the body's core of tumor immunotherapy worker is $\mathrm{T}$ lymphocytes. The role of galectins in immune responses is mainly through regulating levels of activated effector NK cells and T cells. Gal-1 selectively deletes TH1 and TH17 cells and promotes the proliferation of $\mathrm{CD} 4+\mathrm{CD} 25+$ Foxp3+ regulatory $\mathrm{T}$ cells (Tregs), further enhancing immunosuppressive activity. Gal3 can keep the distance between T-cell receptor (TCR) and CD8 molecule, causing the latter inactivation and through inhibiting the interaction between the heavily $O$-glycosylated tumor-derived complex class I-related chain A (MICA) and NK cells, damaging the function of the latter cells, eventually leading to immune evasion. Gal-1 promotes cancer progression through inhibiting immune responses. Perillo et al. [82] found that Gal-1 induced the apoptosis of activated $\mathrm{T}$ cells and their further study [83] revealed that cell surface glycoproteins on activated T cells were the receptors for extracellular Gal-1, such as CD2, T-cell receptor (TCR), and CD95. On the other hand, Gal-1 exposure significantly promotes the differentiation of Treg cells (CD4+CD25+FoxP3+) [84]. Gal-1 treatment in vivo greatly increases IL-10 production inducing $\mathrm{T}$ cells that suppress autoimmune inflammation [85]. Gal-3 was also regarded as one of targeting molecules involved in the immune escape in the progression of cancers [86]. Stillman et al. [87] found that exogenous Gal-3 causes apoptosis in activated T cells. Further study [88] also revealed that Gal-3 suppressed the binding of MHC class I chainrelated molecular, resulting in the impairment of the NK cell activation. Labrie et al. found that Gal-7 expression was induced by mutant p53. Gal-7 not only increased the invasive behavior of ovarian cancer cells by inducing MMP-9 and increasing cell motility but also has immunosuppressive 
properties by killing Jurkat $\mathrm{T}$ cells and human peripheral $\mathrm{T}$ cells [89]. Gonçalves et al. [90] found that a fundamental molecular pathway, which includes ligand-dependent activation of ectopically expressed latrophilin 1 and possibly other G-protein coupled receptors leading to increased translation and exocytosis of the immune receptor Tim-3 and its ligand galectin-9. This occurs in a protein kinase $\mathrm{C}$ and mTOR(mammalian target of rapamycin-) dependent manner. Tim3 participates in galectin- 9 secretion and is also released in a free soluble form. Galectin-9 impairs the anticancer activity of cytotoxic lymphoid cells including natural killer (NK) cells. Soluble Tim-3 prevents secretion of interleukin-2 (IL-2) required for the activation of cytotoxic lymphoid cells. These results were validated in ex vivo experiments using primary samples from acute myeloid leukemia (AML) patients. This pathway provides reliable targets for both highly specific diagnosis and immune therapy of AML (Table 1).

2.6. The Role of Galactins in the Treatment of Cancer. At present, the therapy of cancer mainly concentrated on surgery, radiotherapy, chemotherapy, immunotherapy, and molecular targeted therapy. Studies [91] have shown that hypoxia can significantly reduce the effectiveness of radiation, chemotherapy, and molecular targeted therapy and verified that Gal-1 derived by tumor binding with Nglycans is important in hypoxia and tumor angiogenesis and they also found that hypoxia can induce secretion of Gal-1 by Kaposi's sarcoma cells via NFkB signal pathway. Zhao et al. [92] also showed that the expression of Gal1 was also upregulated by the level of HIF- $1 \alpha$ stabilization within tumors and hypoxia-responsive elements are located at -441 to $-423 \mathrm{bp}$ upstream of the transcriptional start site of the Lgals1 gene and are essential for HIF-1-mediated galectin-1 expression. Other studies [93] also revealed that hypoxia-exposed cancer cells produce the higher levels of Gal-1, which was closely related to HIF- $1 \alpha$ and carbonic anhydrase IX (CA IX) and knocking down Gal-1 can reduce hypoxia-induced invasion and migration of cancer cells. The studies also showed that the increased Gal-1 by hypoxia is related to poor prognosis of cancer patients. The similar study [94] found that Gal-3 was also upregulated within hypoxic regions of murine and human melanomas. Currently, molecular targeted therapy mainly focused on tumor angiogenesis or intratumoral hypoxia-induced protumorigenic signaling. Many research $[95,96]$ have demonstrated that using antiangiogenic drugs could improve the outcomes of chemotherapy or immunotherapy. The studies [97, 98] investigated that the treatments combining Gal-1 inhibition with other cancer therapies showed that anginex (a novel angiogenesis inhibitor) could improve radiochemotherapy outcomes in transgenic model of aggressive breast cancer and A squamous cell (SCCVII) xenograft tumor mouse model, but the detailed mechanism involved was still in research. Croci et al. [79] showed that vessels in anti-VEGF-sensitive tumors express more $\alpha 2-6$-linked sialic acid reducing the secretion of Gal-1. In contrast, anti-VEGF refractory tumors secreted more galectin-1. Garín et al. [84] found that using MMP inhibitors targeting on cleavage of Gal-3 significantly reduced angiogenesis in breast cancer. Grosset et al. [99] found that cytosolic galectin-7 impaired p53 functions and induced chemoresistance in breast cancer cells by affecting mitochondrial transport. Matsukawa et al. [100] used 68 clinical tissues from 18 patients with oral squamous cell carcinoma (OSCC) who received radiotherapy and chemotherapy followed by surgery and detected the expression of galectin7 by proteomic analysis and immunohistochemical analysis. They found that the sensitivity and specificity of the galectin7 prediction score (G7PS) in predicting this resistance were of $96.0 \%$ and $39.5 \%$, respectively, in the 68 test cases. The cumulative 5-year disease-specific survival rate was $75.2 \%$ in patients with resistant prediction using G7PS and 100\% in patients with sensitive prediction. They found that low galectin-7 expression is more likely to exhibit chemotherapy and/or radiotherapy resistance, suggesting that galectin-7 is a potential predictive marker of chemotherapy and/or radiotherapy resistance in patients with OSCC. However, the exact mechanism is still unclear (Table 1).

\section{Galectins in Cervical Cancer}

The current research about the relationship between cervical cancer and galectins mainly concentrated on tumor formation, angiogenesis, radiation, chemotherapy sensitivity, and so on [101, 102]. Gal-1, Gal-3, Gal-7, and Gal-9 have been reported in cervical cancer.

3.1. Galectin-1 in Cervical Cancer. Gal-1 has been shown to be involved in different steps of cancer cell invasion and metastasis by regulating cell adhesion and cell migration [103]. In ovarian and prostate cancer cell lines, Gal-1 promotes the adhesion of tumor cells to the extracellular matrix [104]. Kohrenhagen et al. [105] examined the expression of galectin-1 that was examined in 80 formalin-fixed, paraffinembedded cervical tissues: 20 benign cervical specimens, 20 low-grade squamous intraepithelial lesions (LGSIL), 20 high-grade squamous intraepithelial lesions (HGSIL), and 20 invasive squamous cell carcinomas (ISCC). They found that the intensity of the galectin-1 expression on stromal cells next to the transformed cells increased according to the pathologic grade: benign cervical tissue, LGSIL, HGSIL, and ISCC. The epithelial cells were always negative for galectin-1. These results suggest that galectin-1 expression on stromal cells increases with the histopathologic grade of cervical tissues. In the study of Kim et al. [106], immunohistochemical analysis revealed that galectin 1 expression was found in most peritumoral stroma samples $(72 / 73 ; 98.6 \%)$. Galectin 1 expression was significantly correlated with the depth of invasion in the cervix and lymph node metastasis on univariate analysis. When progression-free survival of all of the patients studied was analyzed based upon galectin 1 expression, galectin 1 expression was not correlated with progression-free survival. Punt et al. [28] analyzed immunohistochemistry combined with clinical data of 155 patients (including 41 relapses and 30 deaths) and found that, as an independent predictor, strong Gal-1 expression was closely related to invasion and metastasis in cervical cancer and an independent predictor for poor survival and a likelihood of receiving postoperative radiotherapy. The functional effects of gal-1 on cervical cancer 
TABLE 2: Effect of galectin-1, galectin-3, galectin-7, and galectin-9 on cervical cancer tissue, cell function, HPV infection, and treatment.

\begin{tabular}{|c|c|c|c|c|}
\hline Process & Galectin-1 & Galectin-3 & Galectin-7 & Galectin-9 \\
\hline Expression in cervical cancer tissues & $\uparrow$ & $\uparrow$ & $\downarrow$ & $\downarrow$ \\
\hline Proliferation & $\uparrow$ & $\uparrow$ & $\downarrow$ & UNK \\
\hline Invasion & $\uparrow$ & $\uparrow$ & $\uparrow$ & UNK \\
\hline HPV infection & UNK & UNK & UNK & $\downarrow$ \\
\hline Radiotherapy and/or chemotherapy resistance & $\uparrow$ & $\uparrow$ & $\downarrow$ & UNK \\
\hline
\end{tabular}

UNK, unknown; $\uparrow:$ increase; $\downarrow$ : decrease.

cells are mainly characterized by promoting cell proliferation and invasion. Kim et al. [106] downregulation of galectin 1 using small interfering RNA resulted in the inhibition of cell growth and proliferation of HeLa and SiHa cells. Moreover, the ability of cells to invade was significantly reduced by galectin-1 small interfering RNA. They chose $\mathrm{HeLa}$ and $\mathrm{SiHa}$ cells to do the galectin 1 small interfering RNA because in both cell lines galectin 1 was overexpressed. Gal1 also has an effect on the radiotherapy and chemotherapy of cervical cancer. Huang et al. [107] revealed that Gal-1 is an independent prognostic factor associated with local recurrence in cervical cancer patients undergoing definitive radiation therapy. Gal-1 also plays an important role in the radiation therapy. The same group in further studies [108] found that Gal-1 mediates radioresistance mainly through two factors: The first factor is DNA repair. Parliament and Murray [109] reviewed the clinical evidence of numerous DNA repair genes involving radioresistance. $\mathrm{H}$-Ras involves DNA repair and radioresistance [110]. In addition, galectin1 can interact with $\mathrm{H}$-Ras to promote downstream signals [111]. The second factor is tumor hypoxia. Hypoxia is a radioresistance-influencing factor for patients with cervical cancer. Coincidentally, hypoxia is associated with galectin1 expression [92]. These findings suggest that Gal-1 mediates radioresistance through the $\mathrm{H}$-Ras-dependent pathway involved in DNA damage repair and targeting Gal-1 may improve the local control of cervical cancer (Table 2).

3.2. Galectin-3 in Cervical Cancer. Galectin-3 plays a role in a variety of physiological and pathological processes. Studies of pancreatic [112], gastric carcinomas [113] found galectin-3 to be upregulated in these tissues compared to normal tissues. Studies conducted by Schoeppner et al. [114] also revealed that galectin-3 expression is related to neoplastic transformation and progression towards metastasis in colon carcinoma. Lee et al. [115] showed that Gal-3 was downregulated in cervical cancer tissues compared to normal tissues and gradually decreased in accordance with the histopathologic grades from LSIL (low-grade squamous intraepithelial lesions) to HSIL (high-grade squamous intraepithelial lesions) and to ISCC (invasive squamous cell carcinomas). Punt et al. [28] found that weak and positive tumor cell galectin-3 expression was correlated with increased and decreased tumor invasion. Study conducted by Balasubramanian et al. [116] through using antigalectin-3 based ELISA and agglutination assays detected galectin-3 level of stages I-V of cervical cancer and found that stage dependent expression of galectin-3 approx. ranging from 1.0 to $3.3,4.4$ to $5.4,5.4$ to $24.7,13.1$ to 31.9 , and 13.9 to $32.9 \mathrm{ng} / \mathrm{mg} \mathrm{C}$ (creatinine) for stages I-V, respectively, indicating that galectin-3 is closely related to the stage of cervical cancer and galectin-3 may be used as a potential diagnostic tool for monitoring or follow-up of the stage of cancer disease. The research about the effect of Gal-3 on the function of cervical cancer cells is little. Study of Liu et al. [117] using cervical carcinoma cell line SiHa cells, through silencing of Gal-3 expression with specific siRNA largely impaired VEGF-C-enhanced cell invasion, indicating that VEGF-C enhanced cervical cancer invasiveness via upregulation of Gal-3 protein through NF- $\kappa \mathrm{B}$ pathway, which may shed light on potential therapeutic strategies for cervical cancer therapy (Table 2).

3.3. Galectin-7 in Cervical Cancer. As an endogenous lectin, a fraction of Gal-7 is constitutively localized at the mitochondria. It has been found to interact with the antiapoptotic protein $\mathrm{Bcl}-2$, suggesting its regulatory role in apoptotic processes [118]. Importantly, increased Gal-7 expression has been shown as a positive predictive biomarker for clinical responses after adjuvant radiation therapy in cervical cancer patients [29]. Zhu et al. [119] detected the expression of Gal7 in normal cervical tissue, CIN I, CIN II, CIN III, and cervical cancer tissues by immunohistochemical method and found that the positive expression rate of Gal-7 in normal cervical tissue, CIN I, CIN II, CIN III, and cervical squamous cell carcinomas were $56.7 \%, 41.9 \%, 32.0 \%, 27.3 \%$, and $25.0 \%$, respectively. The same group also found that the expression of Gal-7 was closely related to the international union of gynecology and obstetrics (FIGO) staging, lymph node metastasis, and 5-year survival rate of cervical squamous carcinoma patients. Higareda-Almaraz et al. [120] found that Gal-7 is downregulated in squamous cervical cancer, highgrade squamous intraepithelial lesions, and cervical cancer cell lines. The results of both researches indicated that Gal7 was low expression in cervical cancer tissues. The research about the effect of Gal-7 on the function of cervical cancer cells is little. Park et al. [102] showed that, in HeLa cells, human cervical epithelial adenocarcinoma cells, Gal-7 could induce matrix metalloproteinase-9 (MMP-9) through p38 MAPK signaling leading to increasing cell invasion and was associated with immunity ability. Gal-7 also has an effect on the radiotherapy and chemotherapy of cervical cancer. Tsai et al. [29] detected different sensitive proteins to radiation and chemotherapy and found that Gal-7 expressed high in the high sensitive group and Gal-7 expressed low in low sensitive 
group, which demonstrated that elevated Gal-7 expression is associated with improved outcomes after radiation therapy for cervical cancer (Table 2).

3.4. Galectin-9 in Cervical Cancer. Gal-9 exhibits lactosebinding activity [121] and is believed to be involved in cellcell or cell-matrix interactions. Gal-9 exhibits a variety of biological functions, such as cell aggregation, adhesion, proliferation, and cell apoptosis and modulation of inflammation [122]. However, the expression of Gal-9 has not been fully verified in human tissues without malignancy as well as malignant tumors. Liang et al. [101] detected the expression of Gal-9 and E-cadherin in normal epithelium and endocervical glands, CIN, and cervical cancer tissues by immunohistochemical method and found that Gal-9 and E-cadherin were evidently detected in normal epithelium and endocervical glands, but those in CIN and SCC were significantly faint. Moreover, both the Gal-9 and E-cadherin expressions in HSIL were significantly lower than those in LSIL, suggesting their association with malignant transformation. Punt et al. [28] found that both galectin-3 and galectin-9 expression were significantly correlated with the presence of HPV type 16 or 18 and tumor expression of galectin- 9 showed a trend towards improved survival (Table 2).

Galectins play an important role in tumorigenicity, the survival of tumor cells, new blood vessels formation, and tumor metastasis. Moreover, it is involved in adjustment of the immune and inflammatory reaction guidance of the tumor escape immune surveillance. Galectin-1, galectin-3, galectin-7, and galectin- 9 may become a prediction to the progression of cervical lesion, valuable markers of prognosis, and basis for target gene therapy in the future.

\section{Conflicts of Interest}

The authors declare that there are no conflicts of interest regarding the publication of this paper.

\section{References}

[1] D. H. Suh, J. W. Kim, M. F. Aziz et al., "Asian society of gynecologic oncology workshop2010," Journal of Gynecologic Oncology, vol. 21, pp. 137-150, 2010.

[2] C. B. J. Woodman, S. I. Collins, and L. S. Young, "The natural history of cervical HPV infection: unresolved issues," Nature Reviews Cancer, vol. 7, no. 1, pp. 11-22, 2007.

[3] J. Y. Kim, S. J. Byun, Y. S. Kim, and J.-H. Nam, "Disease courses in patients with residual tumor following concurrent chemoradiotherapy for locally advanced cervical cancer," Gynecologic Oncology, vol. 144, no. 1, pp. 34-39, 2017.

[4] L. Hertlein, M. Lenhard, A. Kirschenhofer et al., "Cetuximab monotherapy in advanced cervical cancer: A retrospective study with five patients," Archives of Gynecology and Obstetrics, vol. 283, no. 1, pp. 109-113, 2011.

[5] J. E. Kurtz, A.-C. Hardy-Bessard, M. Deslandres et al., "Cetuximab, topotecan and cisplatin for the treatment of advanced cervical cancer: A phase II GINECO trial," Gynecologic Oncology, vol. 113, no. 1, pp. 16-20, 2009.
[6] D. K. Gaffney, K. Winter, A. P. Dicker et al., "A Phase II study of acute toxicity for Celebrex (celecoxib) and chemoradiation in patients with locally advanced cervical cancer: primary endpoint analysis of RTOG 0128," International Journal of Radiational Oncology Biology Physics, vol. 67, pp. 104-109.

[7] F. G. Herrera, P. Chan, C. Doll et al., "A prospective phase III trial of the cyclooxygenase-2 inhibitor celecoxib in patients with carcinoma of the cervix with biomarker assessment of the tumor microenvironment," International Journal of Radiation Oncology Biology Physics, vol. 67, no. 1, pp. 97-103, 2007.

[8] F.-T. Liu and G. A. Rabinovich, "Galectins as modulators of tumour progression," Nature Reviews Cancer, vol. 5, no. 1, pp. 29-41, 2005.

[9] H. Leffler, "Galectins structure and function-a synopsis.", Results and Problems in Cell Differentiation, vol. 33, pp. 57-83, 2001.

[10] H. Leffler, S. Carlsson, M. Hedlund, Y. Qian, and F. Poirier, "Introduction to galectins," Glycoconjugate Journal, vol. 19, no. 7-9, pp. 433-440, 2002.

[11] S. H. Barondes, D. N. W. Cooper, M. A. Gitt, and H. Leffler, "Galectins. Structure and function of a large family of animal lectins," The Journal of Biological Chemistry, vol. 269, no. 33, pp. 20807-20810, 1994.

[12] A. Chhabra and V. Rani, "Gel-Based Gelatin Zymography to Examine Matrix Metalloproteinase Activity in Cell Culture," in Methods Molecular Biology, vol. 1731 of Methods in Molecular Biology, pp. 83-96, Springer, New York, NY, USA, 2018.

[13] S. Saghebasl, S. Davaran, R. Rahbarghazi, A. Montaseri, R. Salehi, and A. Ramazani, "Synthesis and in vitro evaluation of thermosensitive hydrogel scaffolds based on (PNIPAAm-PCLPEG-PCL-PNIPAAm)/Gelatin and (PCL-PEG-PCL)/Gelatin for use in cartilage tissue engineering," Journal of Biomaterials Science-Polymer Edition, vol. 29, no. 10, pp. 1185-1206, 2018.

[14] A. W. Griffioen and V. L. Thijssen, "Galectins in tumor angiogenesis," Annals of Translational Medicine, vol. 2, no. 9, article no. 90, 2014.

[15] K. C. Haudek, K. J. Spronk, P. G. Voss, R. J. Patterson, J. L. Wang, and E. J. Arnoys, "Dynamics of galectin-3 in the nucleus and cytoplasm," Biochimica et Biophysica Acta, vol. 1800, no. 2, pp. 181-189, 2010.

[16] H. Barrow, J. M. Rhodes, and L.-G. Yu, "The role of galectins in colorectal cancer progression," International Journal of Cancer, vol. 129, no. 1, pp. 1-8, 2011.

[17] D. N. W. Cooper and S. H. Barondes, "God must love galectins; he made so many of them," Glycobiology, vol. 9, no. 10, pp. 979984, 1999.

[18] K. Wdowiak, W. Spychałowicz, M. Fajkis, and J. Wojnar, "Galectins in hematological malignancies - role, functions and potential therapeutic targets," Postepy Higieny i Medycyny Doswiadczalnej, vol. 70, pp. 95-103, 2016.

[19] K. D. Johnson, O. V. Glinskii, V. V. Mossine et al., "Galectin-3 as a potential therapeutic target in tumors arising from malignant endothelia," Neoplasia, vol. 9, no. 8, pp. 662-670, 2007.

[20] Y. Zick, M. Eisenstein, R. A. Goren, Y. R. Hadari, Y. Levy, and D. Ronen, "Role of galectin-8 as a modulator of cell adhesion and cell growth," Glycoconjugate Journal, vol. 19, no. 7-9, pp. 517-526, 2002.

[21] Y.-S. Kim, J.-A. Jung, H.-J. Kim et al., "Galectin-3 binding protein promotes cell motility in colon cancer by stimulating the shedding of protein tyrosine phosphatase kappa by proprotein convertase 5," Biochemical and Biophysical Research Communications, vol. 404, no. 1, pp. 96-102, 2011. 
[22] K.-L. Wu, E.-Y. Huang, E.-W. Jhu et al., "Overexpression of galectin-3 enhances migration of colon cancer cells related to activation of the K-Ras-Raf-Erk1/2 pathway," Journal of Gastroenterology, vol. 48, no. 3, pp. 350-359, 2013.

[23] S.-J. Kim, J.-Y. Shin, K.-D. Lee et al., "Galectin-3 facilitates cell motility in gastric cancer by Up-regulating protease-activated receptor-1(PAR-1) and matrix metalloproteinase-1(MMP-1)," PLoS ONE, vol. 6, no. 9, Article ID e25103, 2011.

[24] S. Ueda, I. Kuwabara, and F.-T. Liu, "Suppression of tumor growth by galectin-7 gene transfer," Cancer Research, vol. 64, no. 16, pp. 5672-5676, 2004.

[25] A. Hittelet, H. Legendre, N. Nagy et al., "Upregulation of galectins-1 and -3 in human colon cancer and their role in regulating cell migration," International Journal of Cancer, vol. 103, no. 3, pp. 370-379, 2003.

[26] N. Nagy, H. Legendre, O. Engels et al., "Refined prognostic evaluation in colon carcinoma using immunohistochemical galectin fingerprinting," Cancer, vol. 97, no. 8, pp. 1849-1858, 2003.

[27] L. Gordower, C. Decaestecker, Y. Kacem et al., "Galectin-3 and galectin-3-binding site expression in human adult astrocytic tumours and related angiogenesis," Neuropathology and Applied Neurobiology, vol. 25, no. 4, pp. 319-330, 1999.

[28] S. Punt, V. L. Thijssen, J. Vrolijk, C. D. De Kroon, A. Gorter, and E. S. Jordanova, "Galectin-1, -3 and -9 expression and clinical significance in squamous cervical cancer," PLOS ONE, vol. 10, no. 6, Article ID e0129119, 2015.

[29] C. J. Tsai, E. P. Sulman, P. J. Eifel et al., "Galectin-7 levels predict radiation response in squamous cell carcinoma of the cervix," Gynecologic Oncology, vol. 131, no. 3, pp. 645-649, 2013.

[30] S. Kashiwagi, G. Tsujio, Y. Asano et al., "Study on the progression types of cancer in patients with breast cancer undergoing eribulin chemotherapy and tumor microenvironment," Journal of Translational Medicine, vol. 16, no. 1, 2018.

[31] K. Ito, K. Stannard, E. Gabutero et al., "Galectin-1 as a potent target for cancer therapy: Role in the tumor microenvironment," Cancer and Metastasis Reviews, vol. 31, no. 3-4, pp. 763-778, 2012.

[32] R. R. Braeuer, E. Shoshan, T. Kamiya, and M. Bar-Eli, "The sweet and bitter sides of galectins in melanoma progression," Pigment Cell \& Melanoma Research, vol. 25, no. 5, pp. 592-601, 2012.

[33] G. Elad-Sfadia, R. Haklai, E. Ballan, H.-J. Gabius, and Y. Kloog, "Galectin-1 augments Ras activation and diverts Ras signals to Raf-1 at the expense of phosphoinositide 3-kinase," The Journal of Biological Chemistry, vol. 277, no. 40, pp. 37169-37175, 2002.

[34] G. Elad-Sfadia, R. Haklai, E. Balan, and Y. Kloog, "Galectin3 augments K-ras activation and triggers a ras signal that attenuates ERK but not phosphoinositide 3-kinase activity," The Journal of Biological Chemistry, vol. 279, no. 33, pp. 3492234930, 2004.

[35] F.-T. Liu, R.-Y. Yang, J. Saegusa, H.-Y. Chen, and D. K. Hsu, "Galectins in regulation of apoptosis," Advances in Experimental Medicine and Biology, vol. 705, pp. 431-442, 2011.

[36] V. L. J. L. Thijssen, F. Poirier, L. G. Baum, and A. W. Griffioen, "Galectins in the tumor endothelium: opportunities for combined cancer therapy," Blood, vol. 110, no. 8, pp. 2819-2827, 2007.

[37] H. M. Strik, K. Schmidt, P. Lingor et al., "Galectin-1 expression in human glioma cells: Modulation by ionizing radiation and effects on tumor cell proliferation and migration," Oncology Reports, vol. 18, no. 2, pp. 483-488, 2007.
[38] V. Arcolia, F. Journe, A. Wattier et al., "Galectin-1 is a diagnostic marker involved in thyroid cancer progression," International Journal of Oncology, vol. 51, no. 3, pp. 760-770, 2017.

[39] M. Le Mercier, S. Fortin, V. Mathieu, R. Kiss, and F. Lefranc, "Galectins and gliomas," Brain Pathology, vol. 20, no. 1, pp. 1727, 2010.

[40] O. M. M. Mohafez, M. A. Abd El-Aziz, and A. A. Abd, "ElGhany, The expression pattern of galectin-3 in the rat hepatocellular carcinoma," Bullinof Pharmaceutical Sciences, vol. 35, pp. 97-108, 2012.

[41] R. Y. Ikemori, C. M. L. Machado, K. M. Furuzawa et al., "Galectin-3 up-regulation in hypoxic and nutrient deprived microenvironments promotes cell survival," PLoS ONE, vol. 9, no. 11, Article ID el11592, 2014.

[42] H. Sanchez-Ruderisch, C. Fischer, K. M. Detjen et al., "Tumor suppressor p16INK4a: Downregulation of galectin-3, an endogenous competitor of the pro-anoikis effector galectin1, in a pancreatic carcinoma model," FEBS Journal, vol. 277, no. 17, pp. 3552-3563, 2010.

[43] J. Kopitz, S. André, C. Von Reitzenstein et al., "Homodimeric galectin-7 (p53-induced gene 1) is a negative growth regulator for human neuroblastoma cells," Oncogene, vol. 22, no. 40, pp. 6277-6288, 2003.

[44] K. Polyak, Y. Xia, J. L. Zweier, K. W. Kinzler, and B. Vogelstein, "A model for p53-induced apoptosis," Nature, vol. 389, no. 6648, pp. 300-305, 1997.

[45] F. Bernerd, A. Sarasin, and T. Magnaldo, "Galectin-7 overexpression is associated with the apoptotic process in UVBinduced sunburn keratinocytes," Proceedings of the National Acadamy of Sciences of the United States of America, vol. 96, no. 20, pp. 11329-11334, 1999.

[46] R. Tanikawa, T. Tanikawa, Y. Okada et al., "Interaction of galectin-9 with lipid rafts induces osteoblast proliferation through the c-Src/ERK signaling pathway," Journal of Bone and Mineral Research, vol. 23, no. 2, pp. 278-286, 2008.

[47] C. M. Pfeffer and A. T. K. Singh, "Apoptosis: A Target for Anticancer Therapy," nternational Journal of Molecular Sciences, vol. 19, p. 448, 2018.

[48] Y. Liu and X. Zhu, "Endoplasmic reticulum-mitochondria tethering in neurodegenerative diseases," Translational Neurodegeneration, vol. 6, no. 1, article no. 21, 2017.

[49] J. A. Villa-Pulgarín, C. Gajate, J. Botet et al., "Mitochondria and lipid raft-located FOF1-ATP synthase as major therapeutic targets in the antileishmanial and anticancer activities of ether lipid edelfosine," PLOS Neglected Tropical Diseases, vol. 11, no. 8, Article ID e0005805, 2017.

[50] H. Bao, Q. Zhang, Z. Zhu et al., "BHX, a novel pyrazoline derivative, inhibits breast cancer cell invasion by reversing the epithelial-mesenchymal transition and down-regulating Wnt/ $\beta$-catenin signalling," Scientific Reports, vol. 7, no. 1, article no. $9153,2017$.

[51] M. Hassan, H. Watari, A. AbuAlmaaty, Y. Ohba, and N. Sakuragi, "Apoptosis and molecular targeting therapy in cancer," BioMed Research International, vol. 2014, Article ID 150845, 23 pages, 2014.

[52] J. Lopez and S. W. G. Tait, "Mitochondrial apoptosis: killing cancer using the enemy within," British Journal of Cancer, vol. 112, pp. 957-962, 2015.

[53] S. Zaman, R. Wang, and V. Gandhi, "Targeting the apoptosis pathway in hematologic malignancies," Leukemia \& Lymphoma, vol. 55, no. 9, pp. 1980-1992, 2014. 
[54] F. Lange, B. Brandt, M. Tiedge et al., "Galectin-1 induced activation of the mitochondrial apoptotic pathway: Evidence for a connection between death-receptor and mitochondrial pathways in human Jurkat T lymphocytes," Histochemistry and Cell Biology, vol. 132, no. 2, pp. 211-223, 2009.

[55] C. D. Chung, V. P. Patel, M. Moran, L. A. Lewis, and M. C. Miceli, "Galectin-1 induces partial TCR $\zeta$-chain phosphorylation and antagonizes processive TCR signal transduction," The Journal of Immunology, vol. 165, no. 7, pp. 3722-3729, 2000.

[56] S. Akahani, P. Nangia-Makker, H. Inohara, H.-R. C. Kim, and A. Raz, "Galectin-3: A novel antiapoptotic molecule with A functional BH1 (NWGR) domain of Bcl-2 family," Cancer Research, vol. 57, no. 23, pp. 5272-5276, 1997.

[57] T. Fukumori, Y. Takenaka, N. Oka et al., "Endogenous galectin-3 determines the routing of CD95 apoptotic signaling pathways," Cancer Research, vol. 64, no. 10, pp. 3376-3379, 2004.

[58] N. Mazurek, J. Conklin, J. C. Byrd, A. Raz, and R. S. Bresalier, "Phosphorylation of the $\beta$-galactoside-binding protein galectin-3 modulates binding to its ligands," The Journal of Biological Chemistry, vol. 275, no. 46, pp. 36311-36315, 2000.

[59] S. Moisan, M. Demers, J. Mercier, T. Magnaldo, E. F. Potworowski, and Y. St-Pierre, "Upregulation of galectin-7 in murine lymphoma cells is associated with progression toward an aggressive phenotype," Leukemia, vol. 17, no. 4, pp. 751-759, 2003.

[60] T. Kobayashi, J. Kuroda, E. Ashihara et al., "Galectin-9 exhibits anti-myeloma activity through JNK and p38 MAP kinase pathways," Leukemia, vol. 24, no. 4, pp. 843-850, 2010.

[61] L. Yang, M. Cui, L. Zhang, and L. Song, "FOXM1 facilitates gastric cancer cell migration and invasion by inducing Cathepsin D," Oncotarget, vol. 8, no. 40, 2017.

[62] M. Iiizumi, S. Mohinta, S. Bandyopadhyay, and K. Watabe, "Tumor-endothelial cell interactions: Therapeutic potential," Microvascular Research, vol. 74, no. 2-3, pp. 114-120, 2007.

[63] K. Ito and S. J. Ralph, "Inhibiting galectin-1 reduces murine lung metastasis with increased CD4+ and CD8+ T cells and reduced cancer cell adherence," Clinical \& Experimental Metastasis, vol. 29, no. 6, pp. 561-572, 2012.

[64] A. Banh, J. Zhang, H. Cao et al., "Tumor galectin-1 mediates tumor growth and metastasis through regulation of T-cell apoptosis," Cancer Research, vol. 71, no. 13, pp. 4423-4431, 2011.

[65] A. S. Merseburger, M. W. Kramer, J. Hennenlotter et al., "Loss of galectin-3 expression correlates with clear cell renal carcinoma progression and reduced survival," World Journal of Urology, vol. 26, no. 6, pp. 637-642, 2008.

[66] L. O’Driscoll, R. Linehan, Y. H. Liang, H. Joyce, I. Oglesby, and M. Clynes, "Galectin-3 expression alters adhesion, motility and invasion in a lung cell line (DLKP), in vitro," Anticancer Reseach, vol. 22, no. 6 A, pp. 3117-3125, 2002.

[67] P. Matarrese, O. Fusco, N. Tinari et al., "Galectin-3 overexpression protects from apoptosis by improving cell adhesion properties," International Journal of Cancer, vol. 85, no. 4, pp. 545-554, 2000.

[68] I. Iurisci, N. Tinari, C. Natoli, D. Angelucci, E. Cianchetti, and S. Iacobelli, "Concentrations of galectin-3 in the sera of normal controls and cancer patients," Clinical Cancer Research, vol. 6, no. 4, pp. 1389-1393, 2000.

[69] M. Demers, T. Magnaldo, and Y. St-Pierre, "A novel function for galectin-7: Promoting tumorigenesis by up-regulating MMP-9 gene expression," Cancer Research, vol. 65, no. 12, pp. 5205-5210, 2005.
[70] A. Nobumoto, K. Nagahara, S. Oomizu et al., "Galectin-9 suppresses tumor metastasis by blocking adhesion to endothelium and extracellular matrices," Glycobiology, vol. 18, pp. 735-744, 2008.

[71] T. Kageshita, Y. Kashio, A. Yamauchi et al., "Possible role of galectin-9 in cell aggregation and apoptosis of human melanoma cell lines and its clinical significance," International Journal of Cancer, vol. 99, no. 6, pp. 809-816, 2002.

[72] K. Ito, S. A. Scott, S. Cutler et al., “Thiodigalactoside inhibits murine cancers by concurrently blocking effects of galectin-1 on immune dysregulation, angiogenesis and protection against oxidative stress," Angiogenesis, vol. 14, no. 3, pp. 293-307, 2011.

[73] P. Nangia-Makker, Y. Honjo, R. Sarvis et al., "Galectin-3 induces endothelial cell morphogenesis and angiogenesis," The American Journal of Pathology, vol. 156, no. 3, pp. 899-909, 2000.

[74] A. I. Markowska, F.-T. Liu, and N. Panjwani, "Galectin-3 is an important mediator of VEGF- and bFGF-mediated angiogenic response," The Journal of Experimental Medicine, vol. 207, no. 9, pp. 1981-1993, 2010.

[75] A. I. Markowska, K. C. Jefferies, and N. Panjwani, "Galectin3 protein modulates cell surface expression and activation of vascular endothelial Growth factor receptor 2 in human endothelial cells," The Journal of Biological Chemistry, vol. 286, no. 34, pp. 29913-29921, 2011.

[76] N. D’Haene, S. Sauvage, C. Maris et al., "VEGFR1 and VEGFR2 Involvement in Extracellular Galectin-1- and Galectin-3Induced Angiogenesis," PLoS ONE, vol. 8, no. 6, Article ID e67029, 2013.

[77] E. Aanhane, I. A. Schulkens, R. Heusschen et al., "Different angioregulatory activity of monovalent galectin-9 isoforms," Angiogenesis.

[78] O. Blaževitš, Y. G. Mideksa, M. Šolman et al., "Galectin-1 dimers can scaffold Raf-effectors to increase H-ras nanoclustering," Scientific Reports, vol. 6, Article ID 24165, 2016.

[79] D. O. Croci, J. P. Cerliani, T. Dalotto-Moreno et al., "Glycosylation-dependent lectin-receptor interactions preserve angiogenesis in anti-VEGF refractory tumors," Cell, vol. 156, no. 4, pp. 744-758, 2014.

[80] V. L. Thijssen, G. A. Rabinovich, and A. W. Griffioen, "Vascular galectins: Regulators of tumor progression and targets for cancer therapy," Cytokine \& Growth Factor Reviews, vol. 24, no. 6, pp. 547-558, 2013.

[81] K. Nagahara, T. Arikawa, S. Oomizu et al., "Galectin-9 increases Tim-3+ dendritic cells and CD8+ T cells and enhances antitumor immunity via galectin-9-Tim-3 interactions.," Journal of Immunology (Baltimore, $M d$ : 1950), vol. 181, no. 11, pp. 76607669, 2008.

[82] N. L. Perillo, K. E. Pace, J. J. Seilhamer, and L. G. Baum, "Apoptosis of T cells mediated by galectin-1," Nature, vol. 378, no. 6558, pp. 736-739, 1995.

[83] A. Grigorian, S. Torossian, and M. Demetriou, "T-cell growth, cell surface organization, and the galectin-glycoprotein lattice," Immunological Reviews, vol. 230, no. 1, pp. 232-246, 2009.

[84] M. I. Garín, C.-C. Chu, D. Golshayan, E. Cernuda-Morollón, R. Wait, and R. I. Lechler, "Galectin-1: a key effector of regulation mediated by $\mathrm{CD} 4{ }^{+} \mathrm{CD} 25^{+}$T cells," Blood, vol. 109, no. 5, pp. 2058-2065, 2007.

[85] M. A. Toscano, A. G. Commodaro, J. M. Ilarregui et al., "Galectin-1 suppresses autoimmune retinal disease by promoting concomitant Th2- and T regulatory-mediated antiinflammatory responses," The Journal of Immunology, vol. 176, no. 10, pp. 6323-6332, 2006. 
[86] F. Tomoharu, O. Natsuo, I. Hirofumi, T. Masayuki, and K. Hiro-omi, "Galectin-3 is overexpressed in renal cell carcinoma and it regulates immune suppression through the induction of apoptosis of T cells," Journal of Urology, vol. 179, pp. 91-92, 2008.

[87] B. N. Stillman, D. K. Hsu, M. Pang et al., "Galectin-3 and galectin-1 bind distinct cell surface glycoprotein receptors to induce T cell death," The Journal of Immunology, vol. 176, no. 2, pp. 778-789, 2006.

[88] S. Tsuboi, M. Sutoh, S. Hatakeyama et al., "A novel strategy for evasion of NK cell immunity by tumours expressing core2 Oglycans," EMBO Journal, vol. 30, no. 15, pp. 3173-3185, 2011.

[89] M. Labrie, M. C. Vladoiu, A.-A. Grosset, L. Gaboury, and Y. St-Pierre, "Expression and functions of galectin-7 in ovarian cancer," Oncotarget, vol. 5, no. 17, pp. 7705-7721, 2014.

[90] S. I. Gonçalves, I. M. Yasinska, S. S. Sakhnevych et al., "The Tim-3-galectin-9 Secretory Pathway is Involved in the Immune Escape of Human Acute Myeloid Leukemia Cells," EBioMedicine, vol. 22, pp. 44-57, 2017.

[91] D. O. Croci, M. Salatino, N. Rubinstein et al., "Disrupting galectin-1 interactions with $\mathrm{N}$-glycans suppresses hypoxiadriven angiogenesis and tumorigenesis in Kaposi's sarcoma," The Journal of Experimental Medicine, vol. 209, no. 11, pp. 19852000, 2012.

[92] X.-Y. Zhao, T.-T. Chen, L. Xia et al., "Hypoxia inducible factor-1 mediates expression of galectin-1: The potential role in migration/invasion of colorectal cancer cells," Carcinogenesis, vol. 31, no. 8, pp. 1367-1375, 2010.

[93] Q.-T. Le, G. Shi, H. Cao et al., "Galectin-1: A link between tumor hypoxia and tumor immune privilege," Journal of Clinical Oncology, vol. 23, no. 35, pp. 8932-8941, 2005.

[94] M. Olbryt, A. Habryka, T. Tyszkiewicz et al., "Melanomaassociated genes, MXI1, FN1, and NME1, are hypoxia responsive in murine and human melanoma cells," Melanoma Research, vol. 21, no. 5, pp. 417-425, 2011.

[95] R. K. Shrimali, Z. Yu, M. R. Theoret, D. Chinnasamy, N. P. Restifo, and S. A. Rosenberg, "Antiangiogenic agents can increase lymphocyte infiltration into tumor and enhance the effectiveness of adoptive immunotherapy of cancer," Cancer Research, vol. 70, no. 15, pp. 6171-6180, 2010.

[96] C. S. F. Wong, J. Sceneay, C. M. House et al., "Vascular normalization by loss of Siah2 results in increased chemotherapeutic efficacy," Cancer Research, vol. 72, no. 7, pp. 1694-1704, 2012.

[97] M. Amano, M. Suzuki, S. Andoh et al., "Antiangiogenesis therapy using a novel angiogenesis inhibitor, anginex, following radiation causes tumor growth delay," International Journal of Clinical Oncology, vol. 12, no. 1, pp. 42-47, 2007.

[98] R. P. M. Dings, Y. Yokoyama, S. Ramakrishnan, A. W. Griffioen, and K. H. Mayo, "The designed angiostatic peptide anginex synergistically improves chemotherapy and antiangiogenesis therapy with angiostatin," Cancer Research, vol. 63, no. 2, pp. 382-385, 2003.

[99] A.-A. Grosset, M. Labrie, D. Gagné et al., "Cytosolic galectin7 impairs p53 functions and induces chemoresistance in breast cancer cells," BMC Cancer, vol. 14, no. 1, article no. 801, 2014.

[100] S. Matsukawa, K.-I. Morita, A. Negishi et al., "Galectin-7 as a potential predictive marker of chemo- and/or radio-therapy resistance in oral squamous cell carcinoma," Cancer Medicine, vol. 3, no. 2, pp. 349-361, 2014.

[101] M. Liang, M. Ueno, S. Oomizu et al., "Galectin-9 expression links to malignant potential of cervical squamous cell carcinoma," Journal of Cancer Research and Clinical Oncology, vol. 134, no. 8, pp. 899-907, 2008.
[102] J. E. Park, W. Y. Chang, and M. Cho, "Induction of matrix metalloproteinase- 9 by galectin-7 through p38 MAPK signaling in HeLa human cervical epithelial adenocarcinoma cells," Oncology Reports, vol. 22, no. 6, pp. 1373-1379, 2009.

[103] G. A. Rabinovich, "Galectin-1 as a potential cancer target," British Journal of Cancer, vol. 92, no. 7, pp. 1188-1192, 2005.

[104] J. Ellerhorst, "Differential expression of endogenous galectin-1 and galectin-3 in human prostate cancer cell lines and effects of overexpressing galectin-1 on cell phenotype," International Journal of Oncology, vol. 14, no. 2, pp. 217-224, 1999.

[105] N. Kohrenhagen, H. U. Volker, M. Kapp, J. Dietl, and U. Kammerer, "Increased expression of galectin-1 during the progression of cervical neoplasia," International Journal of Gynecological Cancer, vol. 16, no. 6, pp. 2018-2022, 2006.

[106] H.-J. Kim, I.-G. Do, H.-K. Jeon et al., "Galectin 1 expression is associated with tumor invasion and metastasis in stage IB to IIA cervical cancer," Human Pathology, vol. 44, no. 1, pp. 62-68, 2013.

[107] E.-Y. Huang, C.-C. Chanchien, H. Lin, C.-C. Wang, C.-J. Wang, and C.-C. Huang, "Galectin-1 is an independent prognostic factor for local recurrence and survival after definitive radiation therapy for patients with squamous cell carcinoma of the uterine cervix," International Journal of Radiation Oncology • Biology • Physics, vol. 87, no. 5, pp. 975-982, 2013.

[108] E.-Y. Huang, Y.-F. Chen, Y.-M. Chen et al., "A novel radioresistant mechanism of galectin-1 mediated by $\mathrm{H}$-Ras-dependent pathways in cervical cancer cells," Cell Death \& Disease, vol. 3, no. 1, article e251, 2012.

[109] M. B. Parliament and D. Murray, "Single nucleotide polymorphisms of DNA repair genes as predictors of radioresponse," Seminars in Radiation Oncology, vol. 20, no. 4, pp. 232-240, 2010.

[110] K. A. Cengel, K. R. Voong, S. Chandrasekaran et al., "Oncogenic $\mathrm{K}$-Ras signals through epidermal growth factor receptor and wild-type H-Ras to promote radiation survival in pancreatic and colorectal carcinoma cells," Neoplasia, vol. 9, no. 4, pp. 341348, 2007.

[111] A. Satelli and S. Rao, "Galectin-1 is silenced by promoter hypermethylation and its re-expression induces apoptosis in human colorectal cancer cells," Cancer Letters, vol. 301, no. 1, pp. 38-46, 2011.

[112] P. O. Berberat, H. Friess, L. Wang et al., "Comparative analysis of galectins in primary tumors and tumor metastasis in human pancreatic cancer," Journal of Histochemistry \& Cytochemistry, vol. 49, no. 4, pp. 539-549, 2001.

[113] H.-J. Woo, H.-G. Joo, S.-W. Song, Y.-S. Sohn, and C. Chae, "Immunohistochemical detection of galectin-3 in canine gastric carcinomas," Journal of Comparative Pathology, vol. 124, no. 2-3, pp. 216-218, 2001.

[114] H. L. Schoeppner, A. Raz, S. B. Ho, and R. S. Bresalier, "Expression of an endogenous galactose-binding lectin correlates with neoplastic progression in the colon," Cancer, vol. 75, no. 12, pp. 2818-2826, 1995.

[115] J.-W. Lee, S. Y. Song, J.-J. Choi et al., "Decreased galectin-3 expression during the progression of cervical neoplasia," Journal of Cancer Research and Clinical Oncology, vol. 132, no. 4, pp. 241247, 2006.

[116] K. Balasubramanian, R. Vasudevamurthy, S. U. Venkateshaiah, A. Thomas, A. Vishweshwara, and S. M. Dharmesh, "Galectin-3 in urine of cancer patients: Stage and tissue specificity," Journal of Cancer Research and Clinical Oncology, vol. 135, no. 3, pp. 355363, 2009. 
[117] J. Liu, Y. Cheng, M. He, and S. Yao, "Vascular endothelial growth factor $\mathrm{C}$ enhances cervical cancer cell invasiveness via upregulation of galectin-3 protein," Gynecological Endocrinology, vol. 30, no. 6, pp. 461-465, 2014.

[118] C. Villeneuve, L. Baricault, L. Canelle et al., "Mitochondrial proteomic approach reveals galectin-7 as a novel BCL-2 binding protein in human cells," Molecular Biology of the Cell (MBoC), vol. 22, no. 7, pp. 999-1013, 2011.

[119] H. Zhu, L. Liu, H. Liu et al., "Expression of galectin-7 and S100A9 and development of cervical squamous carcinoma," Journal of Central South University of Technology, vol. 38, pp. 888-895, 2013.

[120] J. C. Higareda-Almaraz, J. S. Ruiz-Moreno, J. Klimentova et al., "Systems-level effects of ectopic galectin-7 reconstitution in cervical cancer and its microenvironment," BMC Cancer, vol. 16, no. 1, article no. 680, 2016.

[121] N. Matsushita, N. Nishi, M. Seki et al., "Requirement of divalent galactoside-binding activity of ecalectin/galectin-9 for eosinophil chemoattraction," The Journal of Biological Chemistry, vol. 275, no. 12, pp. 8355-8360, 2000.

[122] S.-Y. Dai, R. Nakagawa, A. Itoh et al., "Galectin-9 induces maturation of human monocyte-derived dendritic cells," The Journal of Immunology, vol. 175, no. 5, pp. 2974-2981, 2005. 


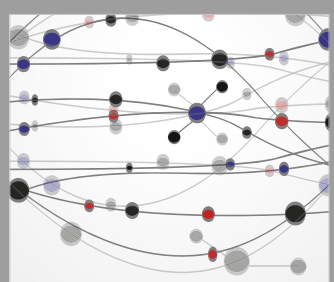

The Scientific World Journal
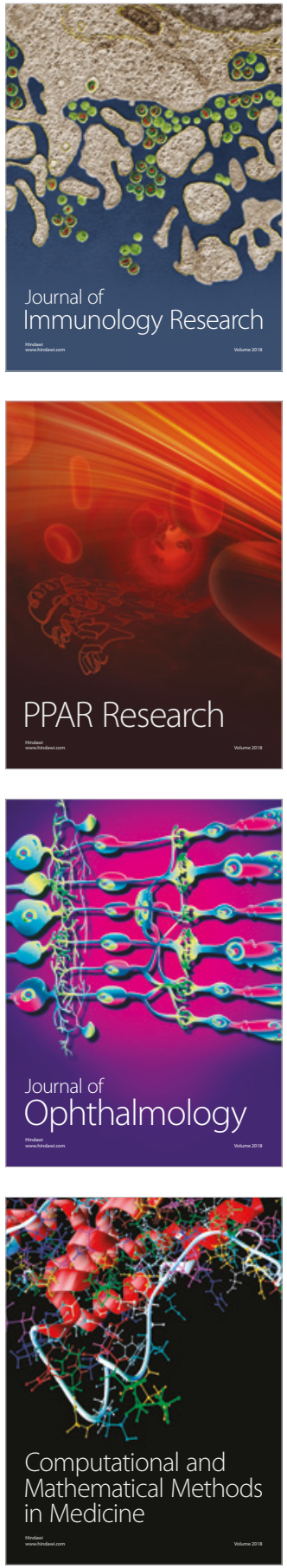

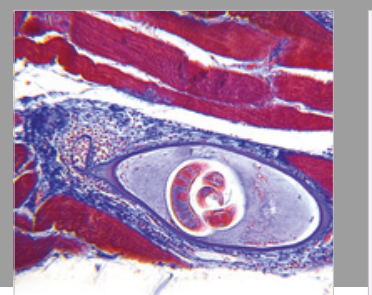

Gastroenterology Research and Practice

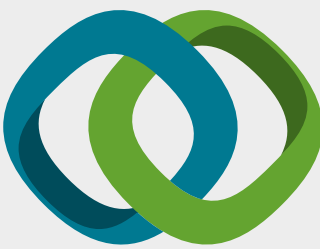

\section{Hindawi}

Submit your manuscripts at

www.hindawi.com
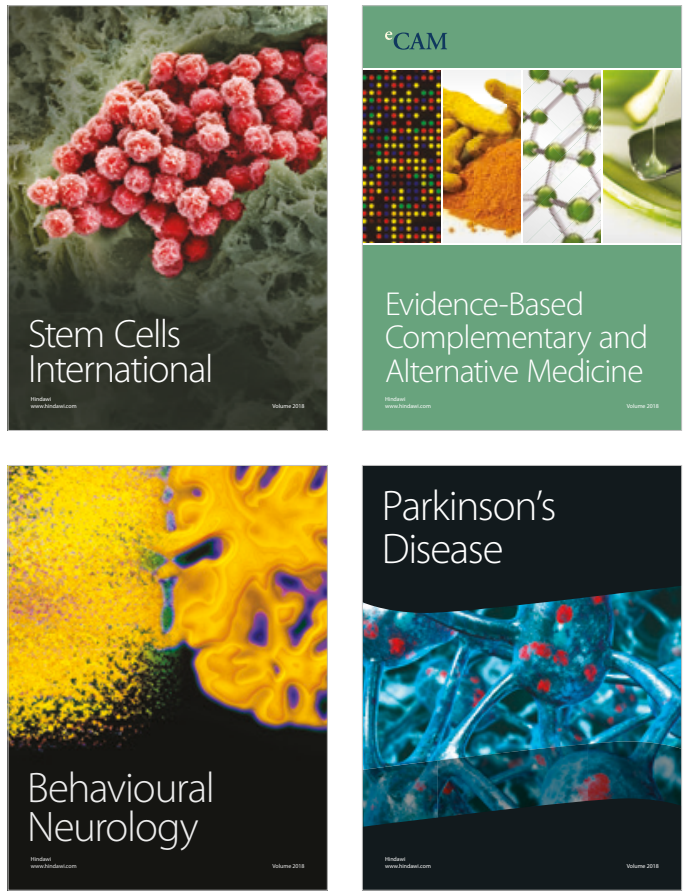

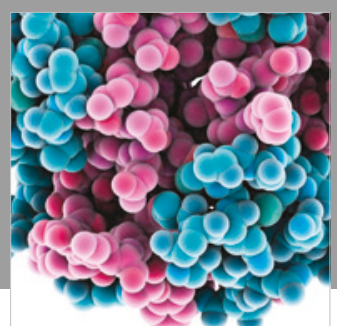

ournal of

Diabetes Research

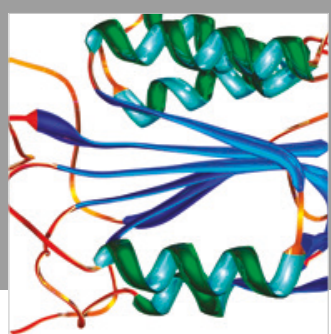

Disease Markers
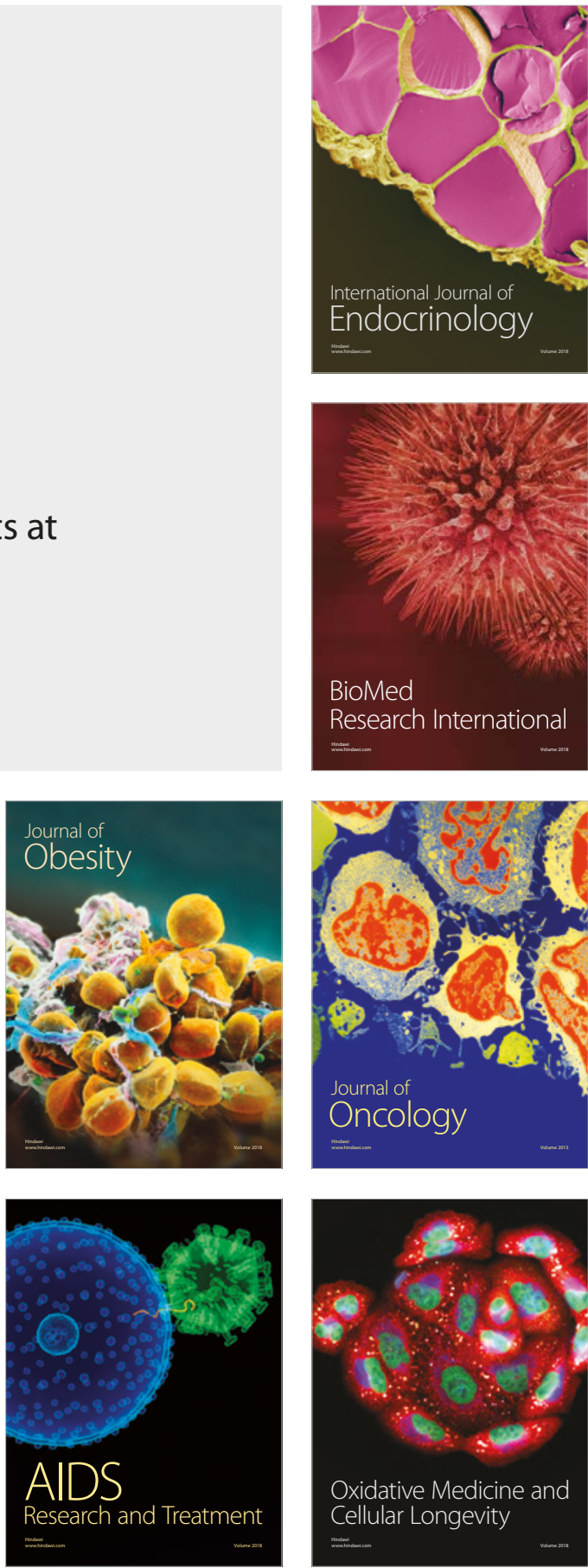